Original Paper http://ajol.info/index.php/ijbcs http://indexmedicus.afro.who.int

\title{
Essais de la substitution partielle du maïs dans l'alimentation par la drêche artisanale séchée sur les performances zootechniques des poulets de chair
}

\author{
S.A. MEBANGA*, K.P. FOOBA et A. MAMOUDOU \\ Ecole des Sciences et de Médecine Vétérinaire - Département de Production Animale, Université de \\ Ngaoundere, B.P: 268 Ngaoundere - Cameroun. \\ *Auteur correspondant ; E-mail : mebengasaaristide@gmail.com
}

\section{RESUME}

L'étude a été menée dans le but d'évaluer les effets du taux d'incorporation de la drêche artisanale séchée sur les performances zootechniques des poulets de chair. A cet effet, 192 poussins de souche Cobb 500 non sexés ont été répartis à partir de la phase de croissance en 4 lots en fonction du taux de substitution du maïs par la drêche artisanale séchée $(0 \%, 15 \%, 30 \%$ et $45 \%)$ pendant 42 jours. Un lot témoin (LT) nourris à base d'une ration contenant le maïs comme principale source d'énergie a été comparée à trois lots expérimentaux (LE) nourris aux rations LE 15, LE 30 et LE 45 dans lesquelles 15, 30 et $45 \%$ de maïs ont remplacés par de la drêche artisanale séchée. Du point de vue pondéral, il n'y a pas eu de différence significative $(p>0,05)$ entre les quatre autres lots. A la $6^{\text {ème }}$ semaine, le poids vif moyen des animaux du LE $45(1416,61 \pm 101,22 \mathrm{~g})$ était significativement $(\mathrm{p}<0,05)$ plus élevé que ceux des autres lots $(1294 \pm 102,06 \mathrm{~g} ; 1232,75 \pm 190,69 \mathrm{~g}$; $1367,57 \pm 210,74 \mathrm{~g})$. La consommation alimentaire était significativement $(\mathrm{p}<0,05)$ élevée chez les poulets des lots LE $30(124,94 \pm 7,3 \mathrm{~g})$ et LE $45(143,35 \pm 9,75 \mathrm{~g})$ par rapport à ceux des lots LT $(109,82 \pm 1,46 \mathrm{~g})$ et LE 15 $(109,66 \pm 7,44 \mathrm{~g})$. Le poids carcasse était significativement $(\mathrm{p}<0,05)$ plus élevé chez les poulets du lot LE 45 $(1065,54 \mathrm{~g})$. Le rendement carcasse ne présentait pas de différence significative $(p>0,05)$ entre les quatre autres lots. Les poids du cœur et du foie étaient significativement $(p<0,05)$ inférieurs dans les lots LT, LE 15 et LE 30 par rapport au lot LE 45. Le prix du kg des aliments a baissé au fur et à mesure que le taux de substitution augmentait. La différence de prix était de 36,7 FCFA entre les lots LT et LE 45. Cependant, le lot LE 30 présentait le meilleur coût de production. En considérant les avantages économiques que présente la drêche artisanale séchée, il apparait en perspective nécessaire de remplacer l'aliment de base qu'est le maïs par ce sous-produit afin de réduire les coûts de production et améliorer la rentabilité des élevages de volaille

(C) 2020 International Formulae Group. All rights reserved.

Mots clés : Taux d'incorporation, drêche, coûts de production, lots expérimentaux, poussins de souche Cobb 500 .

\section{Effect of partial substitution of maize in feed with dried artisanal dreche on the zoo technical performances of broilers}

\begin{abstract}
This study was carried out in order to assess the effects of the rate of dried artisanal dreche on the zoo technical performances of broilers. For this purpose, 192 non-sex cob 500 stock chicks were divided from the growth phase into 4 batches according to the rate substitution of maize by dried artisanal dreche for 42 days. A
\end{abstract}


control batch (LT) fed on a ration containing maize as the main source of energy was compared to three experimental batches (LE 15, LE 30 and LE 45) fed on rations in which 15, 30 and 45\% of maize were been replaced by the dried artisanal dreches. From a weight point of view, there was no significant $(p>0,05)$ difference between the 4 batches. At the $6^{\text {th }}$ week, the average live weight of LE 45 animals $(1416,61 \mathrm{~g})$ was significantly $(\mathrm{p}<0,05)$ higher than those of others batches $(1294 \pm 102,06 \mathrm{~g} ; 1232,75 \pm 190,62 \mathrm{~g} ; 1367,57 \pm 210,74$ g). Feed consumption was significantly $(\mathrm{p}<0,05)$ high in chickens from batches LE 30 and LE 45 compared to those from batches LT and LE 15 . The carcass weight was significantly $(p<0,05)$ higher in chickens from batch LE 45 than in chickens from others batches. The carcass yield showed no significant $(p>0,05)$ difference between the four batches. The weights of the heart and the liver were significantly $(p<0,05)$ lower in the LT, LE 15 and LE 30 batches than that of the LE 45 batch. The price per kg of food decreases as the substitution rate increases. The price difference was 36, 75 FCFA between batches LT and LE 45. However, batch LE 30 had the best production cost. Considering the economic advantages of dried artisanal dreche, it appears in perspective necessary to replace the basic food that is corn by this by-product in order to reduce production costs and improve the profitability of poultry farms.

(C) 2020 International Formulae Group. All rights reserved.

Keywords: Incorporation rate, dreche, production costs, experimental batches, Cobb 500 strain chicks.

\section{INTRODUCTION}

Au Cameroun, la production des poulets de chair est estimée 43726218 têtes et 85,9\% des ménages élèvent les poulets traditionnels (poules et coqs de race locale) dont le cheptel est estimé à 18134814 têtes (MINEPIA, 2013). Cette production n'arrive cependant pas à satisfaire la demande sans cesse croissante sur le marché local et la situation est plus précaire à l'approche des périodes dites «de fête » pendant lesquelles la demande a presque toujours raison des efforts des producteurs (Azebaze, 2008). L'aviculture moderne apparait alors comme une solution attractive pour pallier aux besoins accrus en protéines animales.

Aujourd'hui au Cameroun, un nombre de plus en plus croissant d'éleveurs de volailles fabriquent eux-mêmes les aliments pour leurs animaux. Cette pratique est certainement due entre autres à la hausse des prix des aliments de volaille sur le marché local. Le principal intrant utilisé en aviculture moderne en Afrique est le maïs. L'importance du maïs comme céréale de choix réside dans le fait qu'il est dépourvu de tanins (substance anti nutritionnelle) mais aussi en raison de sa plus grande valeur énergétique par rapport aux autres céréales (Azebaze, 2008).

De nos jours, avec la crise alimentaire mondiale combinée aux problèmes énergétiques, le maïs devient de plus en plus sollicité pour l'alimentation humaine et la production de biocarburants d'où son coût élevé et les difficultés d'approvisionnement en aviculture moderne (Azebaze, 2008). Cette situation a conduit à la recherche effrénée des formules de rations réalisables à moindre coût. L'intérêt et les possibilités d'inclure la drêche de brasserie, trop souvent gaspillée en Afrique, dans une alimentation de finition des poulets de chair pourrait pallier à ce problème. A notre connaissance, peu d'études ont été entreprises sur les performances zootechniques obtenues avec une ration à base de drêche artisanale séchée chez les poulets de chair au Cameroun. C'est dans cette optique que nous avons essayé de modifier à travers nos essais expérimentaux, la formule classique contenant du maïs en remplaçant cette matière première par la drêche artisanale séchée. Le but est de savoir quelles sont les répercussions de cette substitution partielle du maïs par la drêche sur les paramètres de production des poules de chair que sont la croissance pondérale, le gain moyen hebdomadaire, l'indice de consommation, le rendement carcasse, le taux de mortalité et le coût de production.

\section{MATERIEL ET METHODES Zone d'étude}

L'étude s'est déroulée durant la période allant du 09 septembre au 20 octobre 2019 en zone périurbaine, dans le $3^{\text {ème }}$ arrondissement 
de la ville de Ngaoundéré, chef-lieu de la région de l'Adamaoua. Elle couvre une superficie de $65000 \mathrm{~km}^{2}$ et se situe entre le $6^{\text {ème }}$ et le $8^{\text {ème }}$ degré de latitude Nord, le $10^{\text {ème }}$ et le $15^{\text {ème }}$ degré de longitude Est. Le climat est de type soudano-guinéen caractérisé par une saison de pluie d'avril à octobre avec un maximum de pluie en août, et une saison sèche de novembre à mars. La moyenne des précipitations annuelles est de $1500 \mathrm{~mm}$ et les températures moyennes oscillent entre $25^{\circ} \mathrm{C}$ et $45{ }^{\circ} \mathrm{C}$. La population est estimée à environ 800000 habitants et l'économie est basée sur l'élevage, l'agriculture, l'apiculture et le commerce (Minepia, 2013).

\section{Matériel animal - Echantillonnage}

Pour l'échantillonnage, 192 poussins de souche Cobb 500 non sexés livrés par la Société de Provenderie du Cameroun (SPC) ont été répartis de manière aléatoire en 4 lots de 48 animaux en fonction du taux de substitution du maïs par la drêche artisanale séchée à $0 \%$, $15 \%, 30 \%$ et $45 \%$ à savoir :

- Lot témoin (LT) : poussins nourris à base d'une ration où le taux de substitution du maïs est de 0\% (ration sans drêche) ;

- Lot expérimental 15\% (LE 15) : poussins nourris à base d'une ration où le taux de substitution du maïs par la drêche artisanale séchée est de $15 \%$;

- Lot expérimental 30\% (LE 30) : poussins nourris à base d'une ration où le taux de substitution du maïs par la drêche artisanale séchée est de $30 \%$;

- Lot expérimental 45\% (LE 45) : poussins nourris à base d'une ration où le taux de substitution du maïs par la drêche artisanale séchée est de $45 \%$.

La densité au démarrage était de 40 poussins $/ \mathrm{m}^{2}$ et de 10 animaux $/ \mathrm{m}^{2}$ en phase de finition.

\section{Conduite des animaux}

Pendant les vingt premiers jours, les poussins étaient nourris à volonté avec deux aliments (Bro mash 1 pour le démarrage du $1^{\text {er }}$ au $14^{\text {ème }}$ jour et Bro mash 2 pour la croissance du $15^{\text {ème }}$ au $20^{\text {ème }}$ jour) fournis par la SPC.
A partir du $21^{\text {ème }}$ jour, les animaux recevaient chacun des rations expérimentales de type croissance-finition pour poulet de chair. Les aliments distribués aux poulets avaient une composition en matières premières telle que présentée dans le Tableau 1.

Les valeurs nutritives des différentes rations expérimentales Bro mash sont représentées dans le Tableau 2.

Le programme de prophylaxie des poulets de chair proposé par la SPC a été appliqué (Tableau 3).

\section{Collecte des données}

La collecte des données et la détermination des paramètres de production ont été effectuées sur les animaux vivants, sur les carcasses et les organes (cœur, foie et gésier).

Dès le début de l'expérimentation, le poids des poussins a été déterminé afin d'avoir une idée sur le poids moyen des poussins à leur réception.

A partir du $21^{\text {ème }}$ jour, pour suivre l'évolution pondérale, les animaux de chaque lot étaient pesés hebdomadairement jusqu'à la $6^{\text {ème }}$ semaine où ils ont été abattus.

Sur les animaux vivants, le poids vif a été mesuré pendant les six semaines, par des pesées hebdomadaires. Les quantités d'aliments distribués dans les différents lots et les refus ont été pesées quotidiennement pour déterminer les quantités consommées par lot.

Sur les animaux abattus au $42^{\text {ème jour, }}$ le poids carcasse a été mesuré après éviscération complète de l'animal et amputation de la tête et des pattes.

Sur les viscères, les poids du cœur, du foie et du gésier ont été pesés à l'aide d'une balance électronique.

Les données collectées ont permis de calculer les paramètres de production que sont le poids vif $(\mathrm{PV})$, le gain moyen hebdomadaire (GMH), l'indice de consommation (IC), le rendement carcasse (RC) et le taux de mortalité (TM).

\section{Analyses statistiques}

La statistique descriptive et l'analyse de variance du modèle linéaire général uni varié 
ont été effectuées avec le logiciel SPSS (version 18,2008 ) pour l'analyse du poids vif $(\mathrm{PV})$, du gain moyen hebdomadaire (GMH), de l'ingéré alimentaire et de l'indice de consommation (IC). Le modèle linéaire général a été utilisé pour tester les effets des facteurs sur les variables, le test SNK (Student Newman
Keules) et Duncan, pour estimer la signification ou l'homogénéité entre les différents sous-ensembles (test de comparaison entre les moyennes). Les différences ont été considérées comme significatives avec un risque d'erreur de $5 \%$.

Tableau 1: Composition en matières premières des aliments distribués aux animaux.

\begin{tabular}{lllll}
\hline Matières premières & LT $(\mathbf{m g})$ & LE15 $(\mathbf{m g})$ & LE30 $(\mathbf{m g})$ & LE45 $(\mathbf{m g})$ \\
\hline Maïs & 74 & 65,4 & 56 & 49,7 \\
Drêches & 0 & 8,6 & 18 & 24,3 \\
Tourteaux arachide & 15 & 15 & 15 & 15 \\
Farine de poisson & 4 & 4 & 4 & 4 \\
Poudre d'os & 0,5 & 0,5 & 0,5 & 0,5 \\
CMV & 5 & 5 & 5 & 5 \\
Huile de palme & 1 & 1 & 1 & 1 \\
Sel & 0,5 & 0,5 & 0,5 & 0,5 \\
Total & 100 & 100 & 100 & 100 \\
Prix kg (FCFA) & 223,69 & 211,13 & 197,41 & 188,21
\end{tabular}

CMV = Complément Minéral Vitaminé, LT = Lot témoin, LE = Lot expérimental.

Tableau 2: Valeurs nutritives des différentes rations expérimentales utilisées dans cette étude.

\begin{tabular}{lcccc}
\hline Matières premières & LT $(\mathbf{m g})$ & LE15 $(\mathbf{m g})$ & LE30 $(\mathbf{m g})$ & LE45 $(\mathbf{m g})$ \\
\hline EM $(\mathrm{kcal} / \mathrm{kg})$ & 2959,9 & 2801,7 & 2628,7 & 2512,8 \\
Protéine $(\%)$ & 17,8 & 17,5 & 17,1 & 16,8 \\
EM/protéine (kcal/kg) & 166 & 160 & 154 & 150 \\
Cellulose brute (\%) & 5,8 & 5,7 & 5,6 & 5,6 \\
Calcium (\%) & 1,2 & 1,2 & 1,1 & 1,1 \\
Phosphore disponible (\%) & 0,68 & 0,72 & 0,76 & 0,79 \\
Lysine (\%) & 0,5 & 0,5 & 0,4 & 0,4 \\
Méthionine (\%) & 0,3 & 0,3 & 0,3 & 0,3 \\
\hline EM = Energie métabolisable, LT = Lot témoin, LE = Lot expérimental. & &
\end{tabular}

EM = Energie métabolisable, $\mathrm{LT}=$ Lot témoin, $\mathrm{LE}=$ Lot expérimental. 
Tableau 3: Prophylaxie sanitaire appliquée aux animaux dans de cette étude.

\begin{tabular}{|c|c|c|}
\hline Age (jour) & Traitements administrés & Noms des produits utilisés \\
\hline $1,2,3$ & Antistress + diurétique & Maxilayer + hepaturyl \\
\hline $4,5,6$ & Antibiotique & Trisulmicyne \\
\hline 7 & $\begin{array}{l}\text { Vaccin Newcastel/Bronchite infectieuse + } \\
\text { Antistress }\end{array}$ & Cevac Bill + Maxilayer \\
\hline $8,9,10$ & Vitamine + oligo-minéraux & Amintotal + Oligophos \\
\hline 12,13 & Antistress + diurétique & Maxilayer + hepaturyl \\
\hline 14 & $\begin{array}{l}\text { Vaccin Newcastle/bronchite infectieuse + } \\
\text { Antistress }\end{array}$ & Cevac Bill + Maxilayer \\
\hline $16,17,18$ & Anticoccidien + oligo-minéraux & Anticox + oligophos \\
\hline $20,21,22$ & Vitamine & Amintotal \\
\hline $25,26,27$ & Antibiotique & Trisulmicyne \\
\hline 29 & Antiparasitaire & Piperazine \\
\hline $30,31,32$ & Anticoccidien + diurétique & Anticox + hepaturyl \\
\hline 34 & Antistress + diurétique & Maxilayer + hepaturyl \\
\hline 35 & $\begin{array}{l}\text { Newcastle/bronchite infectieuse + } \\
\text { Antistress }\end{array}$ & Cevac Bill + Maxilayer \\
\hline $37,38,39$ & Vitamines + oligo-minéraux & Amintotal + Oligophos \\
\hline
\end{tabular}

\section{RESULTATS}

Effets de la substitution partielle du maïs par la drêche artisanale séchée sur les performances de croissance des poulets de chair

Les résultats sur l'évolution pondérale des poulets figurent dans le Tableau 4.

Il ressort de l'analyse de ce tableau qu'à la $3^{\text {ème }}$ semaine, le poids vif moyen des animaux est semblable entre les 4 lots. La croissance pondérale a augmenté de manière régulière aussi bien chez les LT que chez les LE jusqu' à la $5^{\text {ème }}$ semaine. A la $6^{\text {ème }}$ semaine, le poids vif moyen des animaux du LE 45est significativement $(\mathrm{p}<0,05)$ plus élevé que ceux des autres lots. Globalement la substitution partielle du maïs par la drêche artisanale séchée n'a pas influencé de manière significative $(p>0,05)$ le poids vif des animaux. L'absence de différence significative de poids au démarrage de l'essai confirme une bonne mise en lots.
L'évolution du gain moyen hebdomadaire (GMH) des poulets est représentée dans le Tableau 5.

Il ressort de ce tableau qu'à la $4^{\text {ème }}$ semaine de l'expérimentation, la croissance des animaux du LE 45 était plus rapide que celle des autres lots. La situation a chuté dans la $4^{\text {ème }}$ semaine de l'essai même si la différence n'est pas significative $(p>0,05)$ avec les autres lots à la $6^{\text {ème }}$ semaine.

Effets de la substitution partielle du maïs par la drêche artisanale séchée sur la consommation et l'indice de consommation alimentaires des poulets de chair

Les résultats sur la consommation alimentaire sont présentés dans le Tableau 6 .

Il ressort de ce tableau que la consommation alimentaire était significativement $(\mathrm{p}<0,05)$ plus élevée chez les poulets des lots LE 30 et LE 45 par rapport à ceux des lots LT et LE 15. 
Les résultats sur les variations de l'indice de consommation en fonction des semaines sont présentés dans le Tableau 7.

Il ressort de ce tableau que l'indice de consommation $(10,38)$ était significativement $(\mathrm{p}<0,05)$ élevé chez les poulets du lot LE 15 à la $6^{\text {ème }}$ semaine que chez les autres lots. La moyenne de l'IC à la fin de l'expérimentation était significativement $(\mathrm{p}<0,05)$ plus élevée chez les poulets du lot LE $15(5,22)$ que chez les poulets des autres lots.

\section{Effet de la substitution partielle du maïs par} la drêche artisanale séchée sur les caractéristiques de la carcasse et des organes des poulets de chair

Les résultats sur les caractéristiques de la carcasse et des organes sont présentés dans le tableau 8 .

Il ressort de ce tableau que le poids carcasse est significativement $(\mathrm{p}<0,05)$ plus élevé chez les poulets du lot LE 45 que chez les poulets des autres lots. Le rendement carcasse ne présente pas de différence significative entre les quatre lots bien que celui du lot LE 45 soit un peu plus élevé que les autres. Les poids du cœur et $\mathrm{du}$ foie sont significativement $(\mathrm{p}<$ 0,05 ) inférieurs dans les lots LT, LE 15 et LE 30 par rapport à ceux du lot LE 45. Le poids du gésier du lot LE 30 est supérieur à ceux des autres lots mais cette différence n'est pas significative $(p>0,05)$.

Effet de la substitution partielle du maïs par la drêche artisanale séchée sur la mortalité des poulets de chair

Au terme de notre travail, un taux de mortalité de $4 \%$ a été observé pendant la phase de démarrage où les rations expérimentales n'ont pas été utilisées. Pendant la phase expérimentale, le taux de mortalité était de 2,08\% dans les lots LT et LE 15.

Analyse économique de la substitution partielle du maïs par la drêche artisanale séchée des poulets de chair

Le Tableau 9 présente les coûts de production par kilogramme des rations expérimentales calculées sur la base des prix d'achat des diverses matières premières sur le marché local.

Il ressort de ce tableau que le prix du $\mathrm{kg}$ des aliments a baissé au fur et à mesure que le taux de substitution augmentait. La différence de prix était de 12,99 FCFA entre les lots LT et LE 15, de 27,18 FCFA entre les lots LT et LE 30, de 36,7 FCFA entre les lots LT et LE 45. Cependant, le lot LE 30 présentait le meilleur coût de production.

Tableau 4: Effets de la substitution partielle du maïs par la drêche artisanale séchée sur le poids vif des poulets de chair.

\begin{tabular}{lccccc}
\hline Semaine & LT $(\mathbf{m g})$ & LE 15 $(\mathbf{m g})$ & LE 30 $(\mathbf{m g})$ & LE 45 $(\mathbf{m g})$ & P \\
\hline S3 & $585,21 \pm 9,80$ & $558,37 \pm 10,62$ & $571,4 \pm 9,91$ & $557,77 \pm 20,29$ & 0,963115 \\
S4 & $878 \pm 51,03$ & $831,66 \pm 0,66$ & $873,36 \pm 9,54$ & $903 \pm 148,91$ & 0,960800 \\
S5 & $1207,35 \pm 102,35$ & $1157,30 \pm 99,95$ & $1237,50 \pm 0,40$ & $1277,67 \pm 104,10$ & 0,893820 \\
S6 & $1294 \pm 102,35$ & $1232,75 \pm 190,69$ & $1367,57 \pm 21,74$ & $1416,61 \pm 101,22$ & 0,575008 \\
& & & & & \\
\hline Moyenne & $991,09 \pm 323,38$ & $945,02 \pm 311,74$ & $1017,46 \pm 365,1$ & $1038,59 \pm 387$ & 0,871209 \\
& & & & & \\
\hline S = semaine, LT = Lot témoin, LE= Lot expérimental. & & &
\end{tabular}


Tableau 5: Effets de substitution partielle du maïs par la drêche artisanale séchée sur le gain moyen hebdomadaire des poulets de chair.

\begin{tabular}{lccccc}
\hline Semaine & LT $(\mathbf{m g})$ & LE 15 $(\mathbf{m g})$ & LE 30 $(\mathbf{m g})$ & LE 45 $(\mathbf{m g})$ & P \\
\hline S4 & $290,98 \pm 10,06$ & $274,29 \pm 91,07$ & $301,96 \pm 1,10$ & $345,23 \pm 61,38$ & 0,650578 \\
S5 & $327,35 \pm 17,02$ & $325,64 \pm 19,15$ & $384,14 \pm 108,9$ & $374 \pm 14,00$ & 0,354177 \\
S6 & $164,65 \pm 7,81$ & $76,45 \pm 1,26$ & $110,07 \pm 10,06$ & $139,61 \pm 4,30$ & 0,714220 \\
\hline Moyenne & $260,99 \pm 85,39$ & $225,46 \pm 131,5$ & $265,39 \pm 140,6$ & $286,29 \pm 127,8$ & 0,856455
\end{tabular}

$\mathrm{S}=$ semaine, $\mathrm{LT}=$ lot témoin, $\mathrm{LE}=$ Lot expérimental

Tableau 6: Effets de la substitution partielle du maïs par la drêche artisanale séchée sur la consommation alimentaire individuelle des poulets de chair.

\begin{tabular}{lccccc}
\hline Semaine & LT $(\mathbf{m g})$ & LE 15 $(\mathbf{m g})$ & LE 30 $(\mathbf{m g})$ & LE 45 $(\mathbf{m g})$ & P \\
\hline S4 & $108,39 \pm 8,57$ & $101,09 \pm 9,78$ & $120,74 \pm 10,61$ & $134,91 \pm 8,48$ & 0,6234942 \\
S5 & $109,77 \pm 4,95$ & $114,50 \pm 8,99$ & $120,71 \pm 13,22$ & $141,12 \pm 10,64$ & 0,7252130 \\
S6 & $111,31 \pm 5,65$ & $113,39 \pm 10,34$ & $133,37 \pm 4,25$ & $154,03 \pm 7,66$ & 0,8169382 \\
\hline Moyenne & $109,82 \pm 1,46$ & $109,66 \pm 7,44$ & $124,94 \pm 7,3$ & $143,35 \pm 9,75$ & 0,9806940 \\
\hline
\end{tabular}

$\mathrm{S}=$ semaine, $\mathrm{LT}=$ Lot témoin, $\mathrm{LE}=$ Lot expérimental

Tableau 7: Effets de la substitution partielle du maïs par la drêche artisanale séchée sur l'indice de consommation des poulets de chair.

\begin{tabular}{lccccc}
\hline Semaine & LT $(\mathbf{m g})$ & LE 15 $(\mathbf{m g})$ & LE 30 $(\mathbf{m g})$ & LE 45 $(\mathbf{m g})$ & P \\
\hline S4 & $2,98 \pm 1,01$ & $2,82 \pm 0,09$ & $3,10 \pm 0,02$ & $3,09 \pm 0,99$ & 0,708570 \\
S5 & $2,35 \pm 0,18$ & $2,46 \pm 1,19$ & $2,20 \pm 1,10$ & $2,61 \pm 0,74$ & 0,646801 \\
S6 & $4,73 \pm 1,05$ & $10,38 \pm 0,91$ & $8,48 \pm 0,99$ & $7,72 \pm 1,93$ & 0,921584 \\
\hline Moyenne & $3,35 \pm 1,23$ & $5,22 \pm 4,47$ & $4,59 \pm 3,4$ & $4,49 \pm 2,81$ & 0,455308 \\
\hline S = semaine, LT = Lot témoin, LE= Lot expérimental & & &
\end{tabular}

Tableau 8: Effets de la substitution partielle du maïs par la drêche artisanale séchée sur le poids carcasse, le rendement carcasse et le poids de quelques organes des poulets de chair.

\begin{tabular}{lccccc}
\hline Variable & LT $(\mathbf{m g})$ & LE 15 $(\mathbf{m g})$ & LE 30 $(\mathbf{m g})$ & LE 45 $(\mathbf{m g})$ & P \\
\hline PC $(\mathrm{g})$ & $954,34 \pm 34,67$ & $900,28 \pm 20,14$ & $1019,25 \pm 3,59$ & $1069,54 \pm 21,64$ & 0,832884 \\
RC $(\%)$ & $73,75 \pm 1,75$ & $73,03 \pm 2,43$ & $74,53 \pm 2,45$ & $75,5 \pm 1,65$ & 0,658060 \\
Cœur $(\mathrm{g})$ & $7,6 \pm 0,89$ & $7,8 \pm 0,84$ & $8,2 \pm 1,3$ & $8,6 \pm 0,89$ & 0,836459 \\
Foie $(\mathrm{g})$ & $33,6 \pm 2,3$ & $31,8 \pm 3,77$ & $32,6 \pm 4,88$ & $38,6 \pm 7,86$ & 0,884701 \\
Gésier $(\mathrm{g})$ & $36,6 \pm 4,62$ & $34,20 \pm 1,30$ & $38,6 \pm 2,3$ & $37,20 \pm 2,77$ & 0,980130 \\
\hline PC $=$ Poids carcasse, & RC $=$ rendement carcasse, LT $=$ Lot témoin & LE $=$ Lot expérimental & &
\end{tabular}

$\mathrm{PC}=$ Poids carcasse, $\mathrm{RC}=$ rendement carcasse, $\mathrm{LT}=$ Lot témoin, $\mathrm{LE}=$ Lot expérimental 
Tableau 9: Coûts des matières premières et des rations alimentaires expérimentales chez les poulets de chair.

\begin{tabular}{llllll}
\hline \multirow{2}{*}{ Ingrédients } & $\begin{array}{l}\text { Prix } \\
(\text { Fcfa/kg) }\end{array}$ & \multicolumn{4}{c}{ Rations $(\mathbf{m g})$} \\
& & LT & LE15 & LE30 & LE45 \\
\hline Maïs & 156 & 11544 & 10202 & 8736 & 7753 \\
Drêches artisanales & 05 & 0 & 43 & 90 & 121,5 \\
Tourteaux d'arachide & 250 & 3750 & 3750 & 3750 & 3750 \\
Farine de poisson & 500 & 2000 & 2000 & 2000 & 2000 \\
Poudre d'os & 250 & 125 & 125 & 125 & 125 \\
Huile de palme & 700 & 700 & 700 & 700 & 700 \\
CMV & 850 & 4250 & 4250 & 4250 & 4250 \\
Prix de 100 kg d'aliment (Fcfa) & & 22369 & 21070 & 19651 & 18699 \\
Coût de production (Fcfa) & & 540,125 & 457,127 & 445,623 & 468,4 \\
\hline
\end{tabular}

\section{DISCUSSION}

$\mathrm{Au}$ travers de cette étude, il est intéressant de constater que l'incorporation de drêche artisanale séchée en remplacement partiel du maïs dans l'aliment, induit chez les poulets de chair une augmentation des performances de croissance. Les poulets des 4 lots croissent pratiquement à la même allure. A partir de la $4^{\text {ème }}$ semaine, la croissance pondérale des poulets du LE 45 s'est accélérée jusqu'à la fin de l'expérimentation avec un écart maximal par rapport au LT à la $6^{\text {ème }}$ (1294g contre 1416,61g); les valeurs des LE étant alors constamment supérieures à celles du LT. Cette augmentation de performances serait liée à celle de la consommation alimentaire et à la bonne conversion de l'aliment quand la proportion de drêche augmente. Ceci est en conformité avec les travaux de Tendonkeng (2009) qui ont étudié l'influence de la substitution du tourteau de soja par la farine des feuilles de Moringa oleifera dans la ration de finition du poulet de chair au Cameroun. Ces résultats sont par contre supérieurs à ceux rapportés par Sintondji (1990) qui a étudié l'influence des drêches de brasserie séchées sur l'alimentation des poulets de chair au Benin et a obtenu pour la même période un poids de $727,4 \mathrm{~g}$ pour le LT (nourris sans drêche) et de $776,31 \mathrm{~g}$ pour ceux nourris à base de drêche de brasserie séchée incorporée à 30\%. Nos résultats sont cependant inférieurs à ceux de Tonfack (2017) qui a substitué partiellement le maïs $(0 \%, 5 \%, 15 \%, 25 \%)$ par le son de maïs dans l'alimentation des poulets de chair à Ngaoundéré au Cameroun.

Aucune différence significative ( $p$ > $0,05)$ du gain moyen hebdomadaire $(\mathrm{GMH})$ n'a été observée entre les lots. Ces résultats sont similaires à ceux obtenus par Gongnet (1996) qui a comparé la valeur nutritive du maïs et du sorgho dans l'alimentation des poulets de chair en zone tropicale et inférieurs à ceux de Ngueba (2006) qui a étudié l'influence de la substitution du maïs par le niébé sur les performances zootechniques des poulets de chair au Sénégal. Cela pourrait être dû aux valeurs nutritives du niébé qui sont plus élevées par rapport à celles des drêches artisanales séchées. 
La consommation alimentaire individuelle pendant toute la période d'essai montre une différence significative $(\mathrm{p}<0,05)$ entre les différents lots. La consommation élevée des LE 30 et LE 45 pourrait s'expliquer par le gaspillage et aussi par la diminution du niveau d'énergie dans leurs rations d'où l'ingestion d'une grande quantité d'aliments pour compenser l'insuffisance d'énergie. Ces résultats se rapprochent de ceux d'Azebaze (2008) qui rapporte une consommation plus élevée pour le lot d'animaux nourris au sorgho par rapport à ceux nourris au maïs.

Aucune différence significative ( $\mathrm{p}>$ $0,05)$ n'a été constatée quant à l'indice de consommation (IC). Le meilleur indice de consommation reste celui du lot témoin. Ceci signifie que l'apport de drêche artisanale séchée dans la ration, n'améliore pas l'indice de consommation par rapport à une alimentation classique (sans drêche). Ces résultats sont supérieurs à ceux obtenus par Azebaze (2008). Ceci peut être dû au taux élevé de cellulose dans notre ration expérimentale.

S'agissant du poids carcasse, les LE 30 et LE 45 ont présenté les meilleurs poids carcasse. Cela pourrait s'expliquer par le fait que les poulets nourris au maïs (LT) ont consommé moins d'aliments donc moins de protéines que ceux recevant des drêches. Ces résultats corroborent avec ceux obtenus par Sourokou (2014) qui a évalué les performances zootechnico-économiques des poulets de chair cobb 500 nourris aux rations à base de la farine des graines d'Hibiscus sabdariffa au Sénégal.

Aucune différence significative $(\mathrm{p}>$ $0,05)$ n'a été observée en ce qui concerne le rendement carcasse (RC). Ces résultats sont inférieurs à ceux obtenus par Azebaze (2008). Ceci peut s'expliquer par le fait que les drêches sont des résidus du sorgho, et par conséquent, ont des valeurs nutritives inférieures au sorgho.

L'alimentation étant le poste de dépenses le plus important dans la conduite de cet élevage, toute réduction des charges se traduira par une diminution du coût de production. Une estimation du prix du kilogramme de l'aliment de finition à base de drêche artisanale séchée incorporée a conduit à 186,99 FCFA contre 223,69 FCFA pour l'aliment de finitions utilisées et 300 FCFA celui vendu dans le commerce à Ngaoundéré. La comparaison entre ces valeurs permet de dégager un bénéfice de 112,01 FCFA par kg entre l'aliment à base de drêche et celui du commerce, d'où une incidence significative sur le coût final de production.

\section{Conclusion}

Au terme de cette étude qui s'est fixée comme objectifs la substitution partielle du maïs par la drêche artisanale séchée à un taux de $45 \%$ dans l'alimentation des poulets de chair à l'effet de connaître les répercussions sur les paramètres de production, l'analyse globale des résultats obtenus nous a montré : une accélération de la croissance, une baisse de la consommation alimentaire, une baisse de l'indice de consommation, un faible taux de mortalité, une baisse du coût de production. En somme, l'incorporation de la drêche artisanale séchée au taux de $30 \%$ dans la ration conduit à améliorer la productivité et donc la rentabilité de l'élevage du poulet de chair. Qualité en hausse, coût de production en baisse, tels sont les données de cette étude. En perspective, il serait donc souhaitable, devant la rareté croissante du maïs, céréale alimentaire de base, également utilisé pour la fabrication des aliments pour volailles, que la drêche artisanale séchée soit le produit de substitution dans l'alimentation des volailles, pour la production industrielle des poulets de chair destinées à la consommation. Cet objectif s'inscrit dans le cadre de la lutte pour l'autosuffisance alimentaire.

\section{CONFLIT D'INTERETS} d'intérêts.

Les auteurs ne déclarent aucun conflit

\section{CONTRIBUTIONS DES AUTEURS}

Tous les auteurs ont contribué à la prise en charge des matériels didactique, technique et à la rédaction du manuscrit. Tous les auteurs ont lu et approuvé la version finale du manuscrit. 


\section{REMERCIEMENTS}

Les auteurs expriment leurs sincères remerciements aux membres du Groupe d'Initiative Commune (GIC) agro-pastoral "EFFICIENT"' de l'Adamaoua, pour leur assistance précieuse et la mise à notre disposition de leur bâtiment d'élevage. Nos remerciements s'adressent également à la Société de Provenderie du Cameroun de Ngaoundéré $3^{\text {ème }}$ pour la fourniture en aliments malgré un contexte difficile, et particulièrement à M. NDOBGA Marcel qui a veillé sur les animaux durant toute l'expérimentation.

\section{REFERENCES}

Azebaze SP. 2008. Essais de substitution du maïs par le sorgho et effets sur les performances Zootechniques des poulets de chair. Th. Méd. Vét., 13: 103

Gongnet GP, Vias F. 1996. Contribution à l'étude comparée de la valeur nutritive du maïs (Zea mays) et du sorgho (Sorghum vulgare) dans l'alimentation des poulets de chair en zone tropicale. Actes Inst. Agron. Vet. (Maroc), 16 (4) : 23-28.

MINEPIA. 2013. Direction des Services Vétérinaires. Rapport annuel du Service Régional de la Protection Sanitaire de l'Adamaoua, Ngaoundéré, 320p.
Ngueba ML. 2006. L'influence de la substitution du maïs par le niébé sur les performances de croissance du poulet de chair en milieu tropical sec. Th. Méd. Vét., 33: 103.

Sintondji B. 1990. Influence of dried brewers grains on broiler feeding in Benin. Revue Elev. Méd. Vét. Pays Trop., 43 (2): 239241.

DOI: https://doi.org/10.19182/remvt.8862

Sourokou S. 2014. Performances zootechnicoéconomiques des poulets de chair (cob 500 ) nourris aux rations à base de la farine des graines de la variété verte de bissap (Hibiscus sabdariffa) au Sénégal. Th. Méd. Vét., 27: 140.

Tendonkeng F, Boukila B, Beguide A, Pamo TE. 2009. Essai de substitution du tourteau de soja par la farine des feuilles de Moringa oleifera dans la ration finition des poulets de chair. Revue Africaine de Santé et de Production Animales (RASPA), 7(NS): 47-52.

Tonfack VC. 2017. Essai de substitution partielle du maïs par le son de maïs dans la ration finition des poulets de chair: performances zootechniques et incidence financière. Th. Méd. Vét., Ngaoundéré, Cameroun, 131p. 